\title{
Rapid Pacing-Induced Massive Mitral Regurgitation During Transcatheter Aortic Valve Implantation
}

\author{
Juan Caro-Codón, MD; Silvia Valbuena-López, MD; Carlos Álvarez-Ortega, MD;
} Mar Moreno-Yangüela, MD, PhD; Raúl Moreno, PhD, MD

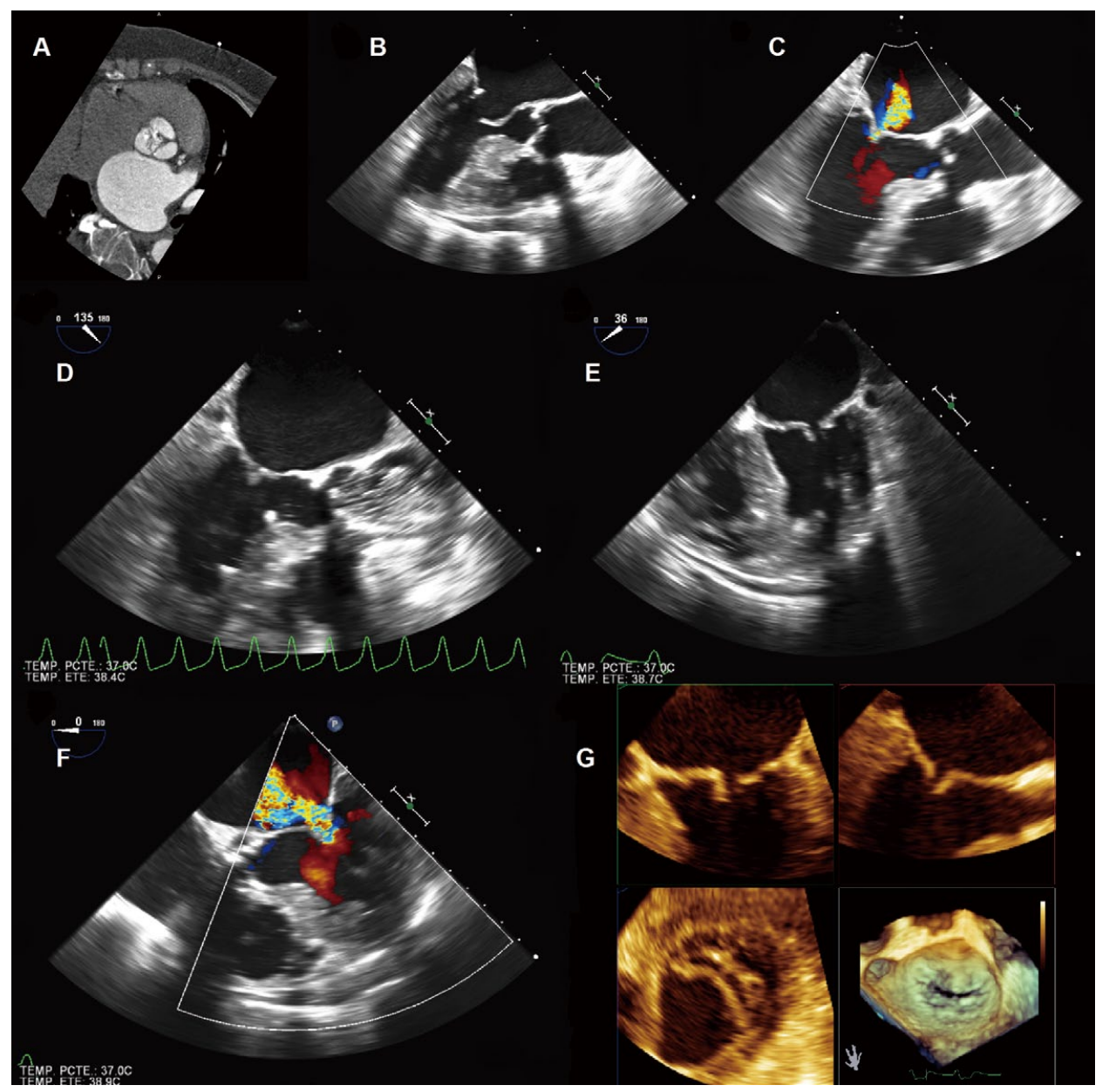

Figure 1. (A) Pre-procedural multislice computed tomography showed significant aortic leaflet calcification. (B) Mid-esophageal $135^{\circ}$ transesophageal echocardiography (TEE) view showed severe aortic stenosis and mild mitral thickening with doming of the anterior leaflet, suggestive of rheumatic disease. (C) Mild mitral regurgitation (MR) without significant stenosis was observed. (D) Long-axis TEE view during balloon aortic valvuloplasty under rapid ventricular pacing. (E) Four-chamber view showing a telesystolic frame with absence of coaptation of both mitral valve leaflets, resulting in (F) severe MR. (G) 3-D multiplanar reconstruction of the mitral valve, showing restricted mobility of both leaflets in telesystole, consistent with a functional mechanism of MR.

Received September 18, 2015; revised manuscript received November 26, 2015; accepted November 29, 2015; released online December 24, 2015 Time for primary review: 25 days

Department of Cardiology, La Paz University Hospital, Madrid, Spain

Mailing address: Juan Caro-Codón, MD, Department of Cardiology, Hospital Universitario La Paz, Paseo de la Castellana 261, Madrid 28046, Spain. E-mail: juancarocd@ gmail.com

ISSN-1346-9843 doi:10.1253/circj.CJ-15-0991

All rights are reserved to the Japanese Circulation Society. For permissions, please e-mail: cj@j-circ.or.jp 


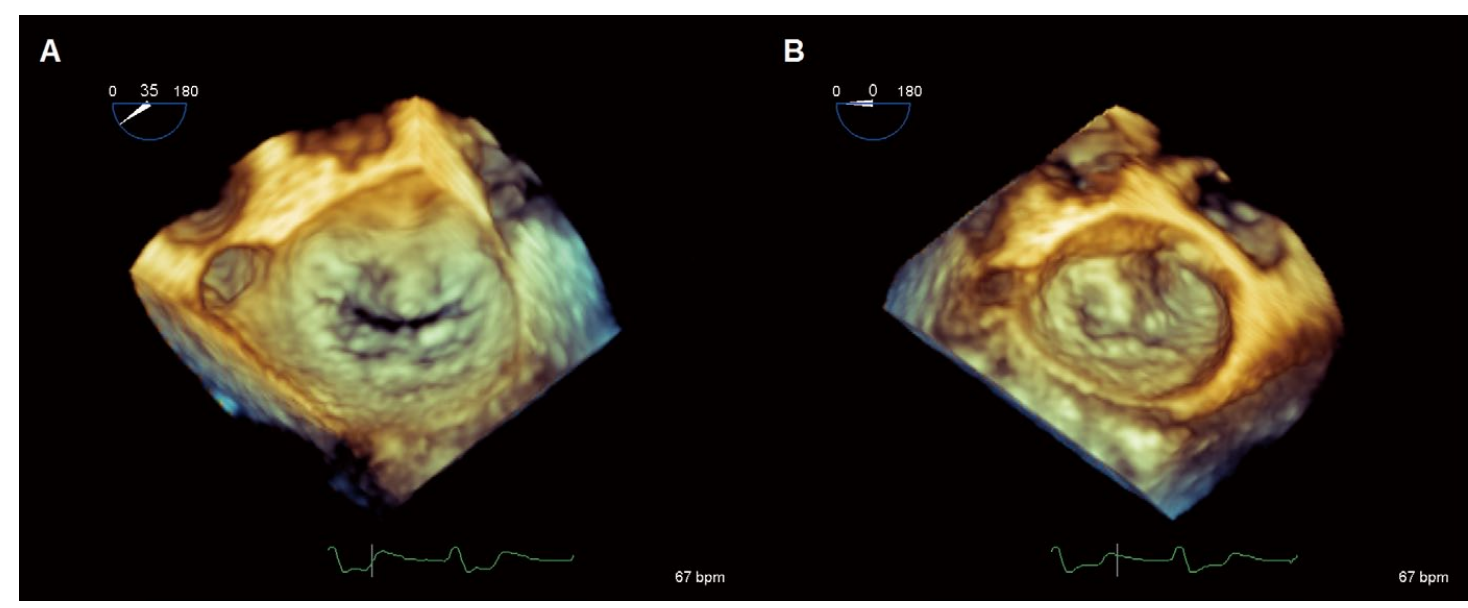

Figure 2. 3-D transesophageal echocardiography (TEE) en face telesystolic view of the mitral valve from the left atrium. After (A) rapid ventricular pacing, severe restriction of the mobility of both mitral leaflets resulted in a large coaptation defect, responsible for massive mitral regurgitation (MR). (B) At the end of the procedure, valve anatomy returned to baseline, with normal leaflet coaptation and only mild MR.

A 91-year-old woman underwent transcatheter aortic valve implantation (TAVI) for symptomatic severe aortic stenosis (Figure 1A). Baseline transthoracic echocardiography showed a severely calcified aortic valve with a medium pressure gradient of $40.5 \mathrm{mmHg}$, an estimated orifice area of $0.73 \mathrm{~cm}^{2}$ and mildly impaired left ventricular (LV) function. A LV ejection fraction of $50 \%$ was calculated using the Simpson biplane method, and there were signs of elevated LV filling pressure (E/E' ratio, 15.7). Pre-procedural evaluation with transesophageal echocardiography (TEE) showed mild mitral valve thickening with doming of the anterior leaflet, consistent with rheumatic valve disease. Mild mitral regurgitation (MR) without significant stenosis was observed (Figures 1B,C). Prior to TAVI placement, balloon aortic valvuloplasty was performed under rapid ventricular pacing (RVP) at 180 beats/min (Figure 1D). Subsequently, also under RVP, a 26-mm balloon-expandable aortic valve (SAPIEN XT, Edwards Lifesciences) was placed. Fluoroscopy and TEE demonstrated optimal valve apposition, without significant paravalvular regurgitation. Nevertheless, the patient had progressive hemodynamic deterioration requiring inotropic support. TEE showed severe biventricular dysfunction and massive MR resulting from restricted systolic motion of both mitral leaflets, leading to incomplete coaptation. Aortic angiography excluded coronary embolization. All wires and catheters were removed from the ventricular cavity in order to rule out any interference with mitral subvalvular apparatus, but severe MR persisted without any change (Figures 1E-G; Movies S1,S2). After several minutes, progressive normalization of LV function took place, and final TEE evaluation confirmed return to baseline situation, with mild MR and normal leaflet coaptation (Figure 2).

Percutaneous aortic valve implantation is an effective and increasingly used technique in patients with severe aortic stenosis. ${ }^{1,2}$ The present case illustrates the deleterious effects that RVP during TAVI can have on myocardial contractility. In this patient, biventricular dysfunction additionally led to functional massive MR, contributing to the hemodynamic instability. La Paz University Hospital is a level 1 cardiac center, wherein 50-60 TAVI procedures are performed each year, and this is the first patient in whom we have observed this "rapid-pacing induced MR" phenomenon. We believe that LV systolic dysfunction prior to TAVI may be a risk factor for this. Limiting RVP time and selection of optimal pacing sites (ie, right ventricular outflow tract) may prevent the development of this severe complication. This case also highlights the critical role of real-time TEE monitoring, which enables the prompt detection and treatment of periprocedural complications. ${ }^{3}$

\section{Disclosures}

The authors declare no conflicts of interest.

\section{References}

1. Fuku Y, Goto T, Komiya T, Sakaguchi G, Shimamoto T, Maruo T, et al. Thirty-day outcome of transcatheter aortic valve implantation with the Edwards SAPIEN XT prosthesis via the transiliofemoral approach: Japanese single-center experience. Circ J 2014; 78: 1357 1363.

2. Sawa Y, Saito S, Kobayashi J, Niinami H, Kuratani T, Maeda K, et al; the MDT-2111 Japan Investigators. First clinical trial of a selfexpandable transcatheter heart valve in Japan in patients with symptomatic severe aortic stenosis. Circ J 2014; 78: 1083-1090.

3. Smith LA, Monaghan MJ. Monitoring of procedures: Peri-interventional echo assessment for transcatheter aortic valve implantation. Eur Heart J Cardiovasc Imaging 2013; 14: 840-850.

\section{Supplementary Files}

Supplementary File 1

Movie S1. Multiplanar reconstruction of the mitral valve confirming restricted mobility of both leaflets, consistent with a functional mechanism of mitral regurgitation.

Supplementary File 2

Movie S2. Four-chamber mid-esophageal view showing biventricular dysfunction and tethered mitral leaflets, with absence of coaptation during systole.

Please find supplementary file(s);

http://dx.doi.org/10.1253/circj.CJ-15-0991 